\title{
National survey on the use of chemicals in the working environment: estimated exposure events
}

\author{
Nanna P Brandorff, Mari-Ann Flyvholm, Inge D Beck, Torsten Skov, Elsa Bach
}

\begin{abstract}
Objectives-To obtain knowledge about the use and distribution of hazardous chemicals in Danish industry. This knowledge is used to regulate the occupational environment and prevent hazardous exposure.

Methods-A national survey on the use of chemicals was carried out in 1989 in a stratified sample of 1448 Danish businesses. 13000 different chemical products were reported. Information on components in the chemical products was obtained from the Danish product register data base (PROBAS) and by inquiries to suppliers and manufacturers. At the end of the study the composition of about 9400 of the products was known. A model was developed to estimate national numbers of chemical exposure events as a supplement to data on weights of chemicals used.
\end{abstract}

Results-Data are presented for 36 chemical substances with chronic toxic effects and high estimated national numbers of exposure events for the industry groups included in the survey. Seven of the 36 substances are carcinogens, 17 are reproductive toxicants, 12 are allergens, and 18 are neurotoxicants according to listings of chronic toxicants used by the Danish authorities. The largest national number of exposure events was estimated for the industry groups manufacture of fabricated metal products, and personal services, cleaning, and hair dressing. These should have special attention in further preventive work.

Working Environment Service,

Landskronagade 3335, DK-2100

Copenhagen, Denmark

N P Brandorff

National Institute of Occupational Health, Lersø Parkallé 105, DK-2100 Copenhagen, Denmark

M-A Flyvholm

I D Beck

T Skov

E Bach

Correspondence to:

Nanna P Brandorff,

Working Environment

Service, Landskronagade

33-35, DK-2100

Copenhagen, Denmark.

Accepted 13 March 1995
Assessment of exposure to chemicals is an important component of both epidemiological studies and setting up priorities for the prevention and regulation of occupational hazards. Information on exposure can be obtained from different sources-for example, measurements of occupational air pollutants, biological monitoring, statistics on production and marketing of chemicals, and reviews and text books on occupational hazards. Measurements of occupational air pollutants and biological monitoring are not practicable for all industries and all chemicals. An alternative method for exposure assessment is based on combining a survey of the uses of chemical products with information on the ingredients of chemical products. ${ }^{12}$

To provide information on the occurrence and use of chemicals in Denmark the Ministry of the Environment and the Ministry of Labour initiated the Danish product register data base (PROBAS) in $1980 .^{3}$ This data base has registrations of hazardous products for occupational use based on mandatory notifications from manufacturers and suppliers. It has been supplemented with information from research and survey projects that include both hazardous and non-hazardous products. ${ }^{3} \mathrm{~A}$ limitation of the PROBAS registration, however, is the lack of systematic registration of products not considered to be hazardous and the lack of detailed and specific information on the exposure (work processes, amount used, number of exposed people, etc).

In a documentation on chemicals that cause chronic health effects (carcinogens, reproductive toxicants, allergens, and neurotoxicants) initiated by the Danish Parliament ${ }^{4}$ the state of knowledge on use, toxic effects, criteria for classification, and the need for further knowledge of these substances was reviewed. It was concluded that further knowledge on the use of and exposure to these chemicals in the Danish working environment was needed and our study was started to obtain such knowledge. Thus the aim of the study was to provide a basic knowledge on the use of and exposure to chemicals in Danish industry with a focus on the chronic toxicants. The study was intended to be the basis for regulatory and voluntary substitution of carcinogens, reproductive toxicants, allergens, and neurotoxicants.

As a supplement to information on the total weight of chemicals used the number of people reported to handle or use the chemicals 
was used in our study to describe the general picture of industrial chemical use in Denmark. To our knowledge, this strategy has not been used before.

Our investigation of a stratified sample of Danish businesses was the first nationwide study of this kind. This paper focuses on potential occupational exposure and presents extrapolated results for selected carcinogens, ${ }^{5}$ reproductive toxicants, ${ }^{6}$ allergens, ${ }^{7-10}$ and neurotoxicants, ${ }^{11}$ defined by listings of chronic toxicants from the Danish authorities or from Scandinavian lists. Other data studied will be published elsewhere.

\section{Methods}

In the first phase of the investigation, initiated in 1989 , data collection was carried out in a stratified sample of 1448 Danish businesses grouped into 19 industrial groups, and four size groups according to the number of employees (unknown, 1-9, 10-99, and $\geqslant 100$ ). The national framework for the survey included about 73000 businesses and 950000 employees. The businesses were asked to complete postal questionnaires of the annual use of chemical products and information about each product on product category (paint, glue, etc), work process (painting, gluing, etc), and the number of people handling or using the products in each process. ${ }^{12}$ In the second phase of data collection, the chemical products reported were identified, and information on components for the products was obtained from PROBAS ${ }^{3}$ and by inquiries to suppliers and manufacturers. ${ }^{13}$ All data received before July 1992 were included.

Chemical products were defined as solid, liquid, or gaseous substances or mixtures of substances. Products were included in this survey when used as: raw materials or semisynthetics in the manufacturing industries; materials for repair, impregnation, or other treatment of machinery, equipment, buildings etc; cleaning agents, disinfectants, or pesticides; materials in administration-for example, photocopy toner.

Chemical substances were defined as chemicals or pure substances identified by a CAS RN (chemical abstract service registry number).

Nineteen industry groups were defined that covered industrial areas with an expected comparable use of chemicals. The appendix lists the type of business and international standard industrial classification (ISIC) $\operatorname{codes}^{14}$ included in each industry group. Industries or businesses with expected low use of chemicals were excluded-that is, several of the subgroups in the wholesale and retail trade; finance, insurance, real estate, and business services; transport, storage, and communication; public administration and defence; recreational and cultural services; teaching; and international and other extraterritorial bodies. Manufacture of food and drink and restaurants and hotels were excluded as the most important chemical products associated with these businesses were considered to be cleaning agents that had been surveyed in an earlier investigation. ${ }^{15}$

To obtain a measure of the number of events that involved possible exposure to the chemicals in question the number of exposure events was defined as the number of products used multiplied by the number of people reported to handle or use the products - that is, one exposure event was defined as one person handling or using one product containing the substance in question. Hence the number of exposure events is a measure of the spread of a chemical.

\section{EXTRAPOLATION MODEL}

The national estimates of the number of exposure events and the national estimates of weights used annually were extrapolated by means of a model based on the assumption that the number of exposure events that involved a chemical substance and the weight of chemical used are proportional to the size of the business measured by the number of employees. In homogenous groups of businesses the total number of exposure events and the total chemical weights were calculated for each substance by the formulas:

$$
\begin{array}{lll}
E=\text { e } A / a & \text { or } & E=\text { e } B / b \\
M=m A / a & \text { or } & M=m B / b
\end{array}
$$

where:

$E=$ total number of exposure events for the chemical for all work sites in the size group within the study framework

$M=$ total weight of the chemical for all work sites in the size group within the study framework

$e=$ number of exposure events for the specific substance among the sample respondents within that size group

$\mathrm{m}=$ weight of chemical used or handled by sample respondents within that size group

$\mathrm{A}=$ number of employees in all work sites in the size group within the study framework $a=$ number of employees in the sample respondent work sites within that size group $B=$ number of work sites within the study framework

$b=$ number of respondent work sites

the figures $B$ and $b$ are chosen for work sites from which information about size was missing.

A calculation of $m$ was made with the following formulas:

$$
\mathrm{m}=\sum_{\mathrm{i}=1}^{\mathrm{n}} \mathrm{k}_{\mathrm{i}} \mathrm{p}_{\mathrm{i}} \quad \text { and } \quad \mathrm{p}_{\mathrm{i}}=\sum_{\mathrm{h}=1}^{\mathrm{n}} \mathrm{p}_{\mathrm{i}, \mathrm{h}}
$$

where: $k_{i}=$ concentration of substance in product number $\mathrm{i}$

$\mathrm{p}_{\mathrm{i}}=$ total weight of the product number $\mathrm{i}$ used by businesses within that size group

$h=$ work site number " $h$ " within that size group.

The national weight of a substance used or handled in a group of businesses was calculated as the sum of all Ms within all the size groups. The national number of exposure events in an industry group was calculated as the sum of all Es within all the size groups. 
ESTIMATION ON SELECTED CHEMICALS

The survey covered in principle all chemical substances registered in the identified products. In accordance with the aims of the study the extrapolated national results for the industry groups included in the survey are presented for carcinogens (C), ${ }^{5}$ reproductive toxicants (R), ${ }^{6}$ allergens (A), ${ }^{7-10}$ and neurotoxicants $(N)^{11}$ as defined from lists of substances with chronic toxic effects used by the Danish authorities or from Scandinavian lists (CRAN substances). Results are presented for substances with chronic toxic effects and with a total of 25000 or more estimated exposure events.

Estimated numbers of exposure events, estimated annual weights used, and numbers of registered products containing the substances were limited to the reported products, for which component information was obtained. All data presented in the text and tables are extrapolated into national figures for the industries included in the survey. All results shown are based on substances defined by CAS RN. In case of discrepancies between the chemical name and the CAS RN, the CAS $R N$ is taken to be the true identification of the substance. This is included in the tables and shown in parentheses when a substance is mentioned in the text for the first time. For white spirit a special CAS RN was constructed for use in PROBAS only (beginning with 99999 ...) that represents white spirit not further specified.

\section{Results}

Answers were received from 1010 of the 1448 businesses (70\%; 438 non-responders). Of these, 894 used chemicals and had employees. About 13000 different chemical products were reported. When the second data collection was closed in July 1992, chemical composition was identified for about 9400 products $(73 \%)$ containing about 4800 different substances. Information on the chemical composition was not available for the remaining 3600 products in July 1992 . For the individual industry groups, information on the component substances was obtained for $66 \%-95 \%$ of the products. The annual weights used were reported for $88 \%$ of the total number of different products. About 4350 products were registered to contain CRAN substances. Reproductive toxicants occurred in $29 \%$ of the products, neurotoxicants in $27 \%$, allergens in $21 \%$, and carcinogens in $16 \%$.

\begin{tabular}{|c|c|c|c|c|c|c|c|c|}
\hline \begin{tabular}{l}
3 \\
3 \\
\multirow{2}{*}{}
\end{tabular} & 芯 & 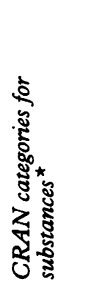 & 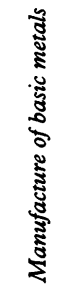 & 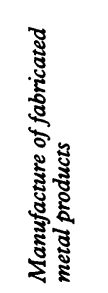 & 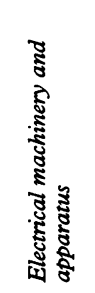 & 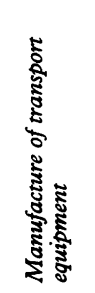 & 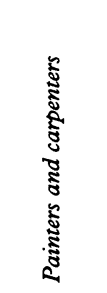 & 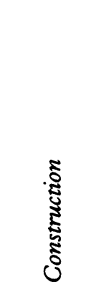 \\
\hline \multicolumn{3}{|c|}{ Industry group number } & 011 & 012 & 013 & 014 & 021 & 022 \\
\hline 71-43-2 & Benzene & CR N & 230 & 16000 & 670 & 3200 & 250 & 5100 \\
\hline $\begin{array}{r}108-46-3 \\
1675-54-3\end{array}$ & $\begin{array}{l}\text { 1,3-Benzenediol } \\
\text { Bisphenol A diglycidylether }\end{array}$ & & 10 & 570 & 80 & $\overline{-1}$ & 1300 & 16 \\
\hline $\begin{array}{l}1675-54-3 \\
1303-96-4\end{array}$ & & ${ }_{R}^{A}$ & $\begin{array}{l}40 \\
31\end{array}$ & $\begin{array}{r}15000 \\
3700\end{array}$ & $\begin{array}{r}1500 \\
95\end{array}$ & $\begin{array}{r}440 \\
6900\end{array}$ & 1500 & 4800 \\
\hline $78-93-3$ & Butanone & $\mathrm{R} N$ & 300 & 12000 & 820 & $\begin{array}{r}6000 \\
360\end{array}$ & 880 & $\begin{array}{r}2000 \\
670\end{array}$ \\
\hline $111-76-2$ & Butoxyethanol & $\mathbf{N}$ & - & 20000 & 1500 & 1900 & 290 & 5800 \\
\hline $141-32-2$ & Butyl acrylate & A & 二 & 6500 & 76 & 110 & 5900 & 4800 \\
\hline $26172-55-4$ & 5-Chloro-2-methyl-3-isothiazolone & A & 二 & 48000 & 8000 & 13000 & 3600 & 14000 \\
\hline $108-94-1$ & Cyclohexanone & $\mathbf{N}$ & 10 & 3900 & 810 & & 000 & 1200 \\
\hline $75-71-8$ & Dichlorodifluoromethane & $\mathbf{N}$ & 190 & 7900 & 1000 & 430 & 1700 & 4400 \\
\hline $75-09-2$ & Dichloromethane & CR N & 660 & 3100 & 460 & 340 & 2100 & 5700 \\
\hline $60-00-4$ & EDTA & $\mathbf{R}$ & 130 & 4100 & 100 & 3700 & 2700 & 1900 \\
\hline $139-33-3$ & EDTA, disodium salt & $\hat{\mathbf{R}}$ & & 12000 & 2600 & 12000 & & 3100 \\
\hline $64-02-8$ & EDTA, tetrasodium salt & $\mathbf{R}$ & 280 & 10000 & 840 & 1500 & 2700 & 4700 \\
\hline 106-89-8 & Epichlorohydrin & CRAN & 40 & 17000 & 2100 & 260 & 1500 & 5000 \\
\hline $141-43-5$ & Ethanolamine & $\mathbf{N}$ & 260 & 10500 & 1900 & 3000 & 290 & 4500 \\
\hline 64-17-5 & Ethyl alcohol & $\mathbf{R}$ & 120 & 20000 & 3100 & 2400 & 6000 & 9800 \\
\hline $75-21-8$ & Ethylene oxide & CR N & 150 & 2000 & 170 & 330 & 15 & 3300 \\
\hline $50-00-0$ & Formaldehyde & $\mathrm{CA}$ & 120 & 20000 & 1200 & 910 & 18000 & 4800 \\
\hline $123-31-9$ & Hydroquinone & RA & - & 900 & 460 & 190 & & 850 \\
\hline 98-82-8 & Isopropylbenzene & $\mathbf{N}$ & - & 11000 & 180 & 210 & 760 & 1200 \\
\hline $108-31-6$ & Maleic acid anhydride & A & 20 & 19000 & 430 & 400 & 2100 & 2300 \\
\hline $80-62-6$ & Methyl methacrylate & RA & 210 & 12000 & 260 & 1600 & 1800 & 2800 \\
\hline $\begin{array}{r}95-70-5 \\
101-68-8\end{array}$ & $\begin{array}{l}\text { 2-Methyl-1,4-benzendiamine } \\
\text { Methylenebisphenylene diisocyanate }\end{array}$ & A & 77 & & & & $700 \overline{0}$ & 7800 \\
\hline $\begin{array}{l}101-80-8 \\
2682-20-4\end{array}$ & $\begin{array}{l}\text { 2-Methyl-3-isothiazolone } \\
\text { - }\end{array}$ & A & II & 48000 & 8000 & 13000 & $\begin{array}{l}7600 \\
3600\end{array}$ & $\begin{array}{r}7800 \\
14000\end{array}$ \\
\hline $108-95-2$ & Phenol & $\mathbf{N}$ & 180 & 8200 & 1400 & 700 & 440 & 4300 \\
\hline $14808-60-7$ & Quartz $\left(\mathrm{SiO}_{2}\right)$ & C & 130 & $\mathbf{7 4 0 0}$ & 50 & 260 & & 12000 \\
\hline \multirow[b]{2}{*}{$64742-88-7$} & \multirow{2}{*}{$\begin{array}{l}\text { Silica (unspecified) } \\
\text { Solvent naphtha (petroleum), } \\
\text { medium aliphatic }\end{array}$} & C & 1400 & 47000 & 3800 & 2500 & 10000 & 18000 \\
\hline & & & & & & 430 & & \\
\hline \multirow{7}{*}{$\begin{array}{r}100-42-5 \\
108-88-3 \\
71-55-6 \\
102-71-6 \\
999999-95-0 \\
1330-20-7\end{array}$} & $\begin{array}{l}\text { medium aliphatic } \\
\text { Styrene }\end{array}$ & RN & $\begin{array}{r}20 \\
110\end{array}$ & 22000 & 1000 & $\begin{array}{l}430 \\
770\end{array}$ & $\begin{array}{l}100 \\
4800\end{array}$ & $\begin{array}{l}3600 \\
4600\end{array}$ \\
\hline & Toluene & RN & 420 & 64000 & 1500 & 2700 & 15000 & 5400 \\
\hline & 1,1,1-Trichloroethane & $\mathbf{N}$ & 880 & 7000 & 3000 & 2600 & 4500 & 2400 \\
\hline & Triethanolamine & A & 710 & 21000 & 1000 & 7400 & 2100 & 14000 \\
\hline & White spirit & RN & 170 & 27000 & 1400 & 3900 & 35000 & 12000 \\
\hline & Xylenes & RN & 790 & 97000 & 4400 & 3500 & 16000 & 15000 \\
\hline & Total & & 7700 & 650000 & 54000 & 92000 & 150000 & 210000 \\
\hline
\end{tabular}

${ }^{\star} \mathrm{C}=$ carcinogenic; $\mathrm{R}=$ reproductivity toxicity; $\mathrm{A}=$ allergenic; $\mathrm{N}=$ neurotoxic. 
The total estimates of the number of exposure events were above or equal to 25000 for a total of 36 substances (table 1). Among these substances seven (19\%) were carcinogens, 17 ( $47 \%)$ were reproductive toxicants, $12(33 \%)$ were allergens, and $18(50 \%)$ were neurotoxicants. For this selected group of substances two additional exposure measures were evaluated: estimated national weights of each substance used in each industry group (table 2) and number of different products containing each chemical substance. ${ }^{\star}$

ESTIMATED NUMBERS OF EXPOSURE EVENTS The highest national estimates of the number of exposure events were found in manufacture of fabricated metal products and personal services, cleaning, and hair dressing. All but one of the 36 substances were used in more than half of the industry groups that were included (table 1). 2-Methyl-1,4-benzendiamine (9570-5) was found in one industry group only, the personal services, cleaning, and hair dressing group, where it is used as a pigment in hair colour preparations. The high estimate of

*A table on number of different products containing each substance is available from the authors. the number of exposure events for this substance may represent a methodological problem originating from the separate registration in PROBAS of nearly identical products, such as hair colour preparations with different shades. The estimated weight of this substance was $44 \mathrm{~kg}$.

The highest national estimates of the number of exposure events for any of the industry groups were obtained for a preservative, the active ingredients in Kathon CG (5-chloro2-methyl-3-isothiazolone

(26172-55-4) (260 000) and 2-methyl-3-isothiazolone (2682-20-4) (250 000)) occurring mainly in personal services, cleaning, and hair dressing and in manufacture of fabricated metal products, and for xylenes (1330-20-7) (220 000) occurring mainly in manufacture of fabricated metal products, and in agriculture, hunting, and forestry; followed by ethyl alcohol (64-17-5) (180 000) occurring mainly in personal services, cleaning, and hair dressing, and in health services and pharmacies; silica (unspecified) (7631-86-9) (180 000) occurring mainly in manufacture of fabricated metal products and in personal services, cleaning, and hair dressing; and ethanolamine (141-43-5) (160 000) occurring mainly in personal services, cleaning, and hair dressing.

\begin{tabular}{|c|c|c|c|c|c|c|c|c|c|c|c|c|c|}
\hline 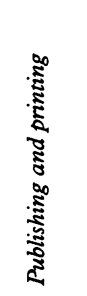 & 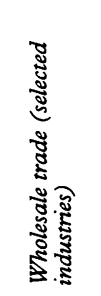 & 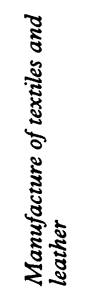 & 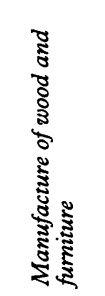 & 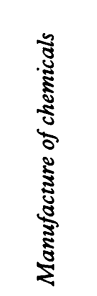 & 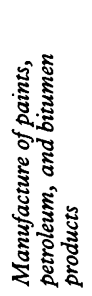 & 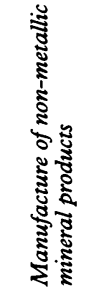 & 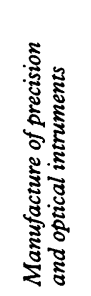 & 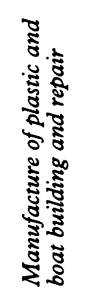 & 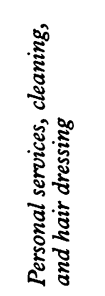 & 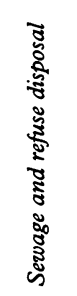 & 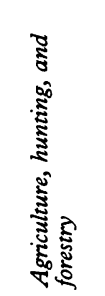 & 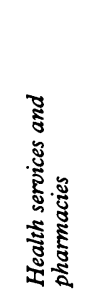 & ڤ్̃ \\
\hline 031 & 041 & 051 & 052 & 053 & 054 & 055 & 056 & 057 & 081 & 082 & 101 & 111 & \\
\hline 2100 & 3900 & 430 & 1400 & 1400 & 210 & 3100 & 46 & 36 & 140 & 30 & 1700 & 560 & 41000 \\
\hline 260 & - & - & - & 39 & - & 140 & - & - & 94000 & - & - & 54 & 96000 \\
\hline 770 & 950 & 380 & 510 & - & - & 1600 & 2300 & 150 & - & - & - & 96 & 30000 \\
\hline 1000 & 560 & 98 & 560 & 490 & - & 150 & 69 & 21 & 540 & 240 & 5200 & 3100 & 25000 \\
\hline 4100 & 1200 & 1300 & 1600 & 270 & - & 680 & 330 & 710 & 260 & 28 & 1500 & 300 & 27000 \\
\hline 5100 & 6000 & 3800 & 1500 & 290 & 1050 & 690 & 78 & 1500 & 3100 & 420 & 740 & 1300 & 55000 \\
\hline 320 & 170 & 12 & 970 & - & 1100 & 100 & 29 & - & 10000 & - & 1300 & 930 & 32000 \\
\hline 20000 & 24000 & 7100 & 20000 & 300 & 460 & 17000 & 52 & 5400 & 52000 & 940 & 670 & 24000 & 260000 \\
\hline 1300 & 260 & 4200 & 280 & 360 & 210 & 36 & 850 & 520 & - & - & 13000 & 24 & 27000 \\
\hline 360 & 1100 & 1300 & 3000 & - & - & 410 & 6 & 1000 & 4000 & - & 540 & 1400 & 29000 \\
\hline 360 & 1900 & 380 & 110 & 8300 & 260 & 510 & 650 & 530 & & - & 2 & 1200 & 27000 \\
\hline 1800 & 1400 & 1800 & 240 & 91 & - & 3000 & 29 & 1900 & 21000 & 39 & 1500 & 7400 & 53000 \\
\hline 760 & 4900 & 820 & 4700 & - & - & 220 & 23 & 1500 & & - & 4 & 2200 & 45000 \\
\hline 9800 & 14000 & 6400 & 2100 & 1700 & 210 & 5900 & 1500 & 580 & 44000 & 850 & 120 & 36000 & 140000 \\
\hline 610 & 1400 & 450 & 510 & - & 38 & 2100 & 1800 & 150 & - & - & - & 44 & 33000 \\
\hline 1050 & 4700 & 820 & 2300 & 7100 & 19 & 140 & - & 950 & 110000 & - & 2200 & 8800 & 160000 \\
\hline 14000 & 7900 & 4900 & 7400 & 18000 & 940 & 5800 & 6500 & 730 & 31000 & 130 & 1400 & 38000 & 180000 \\
\hline 480 & 940 & 1700 & 280 & 450 & - & 400 & 170 & 760 & 21000 & - & 1100 & 11000 & 44000 \\
\hline 7600 & 2000 & 1700 & 4800 & 8100 & 400 & 11000 & 58 & 690 & 30000 & 120 & 8800 & 11000 & 130000 \\
\hline 9700 & 140 & - & 120 & 13 & - & 190 & 2200 & 110 & 32000 & - & 7 & 5700 & 53000 \\
\hline 2000 & 1100 & 60 & 820 & 200 & 930 & 570 & 6 & 120 & 10000 & 4 & 2100 & 2100 & 33000 \\
\hline 880 & 860 & 650 & 660 & 250 & 530 & 150 & 69 & 780 & - & - & - & 76 & 29000 \\
\hline 930 & 1100 & 730 & 270 & 13 & 1300 & 460 & 2200 & 53 & 10000 & 20 & 1300 & 5800 & 43000 \\
\hline- & - & - & - & - & - & - & - & & 94000 & - & - & - & 94000 \\
\hline 2300 & 1800 & 30 & 62 & - & - & 950 & 580 & 1200 & & - & - & 320 & 25000 \\
\hline 17000 & 24000 & 7100 & 20000 & 300 & 460 & 17000 & 29 & 4600 & 52000 & 940 & 670 & 24000 & 250000 \\
\hline 1200 & 840 & 2600 & 670 & 580 & - & 1300 & 1300 & 840 & 260 & 210 & 550 & 1200 & 27000 \\
\hline 360 & 270 & 380 & 400 & 6900 & 610 & 1400 & 110 & 170 & 10000 & - & - & 210 & 41000 \\
\hline 12000 & 13000 & 1900 & 6100 & 1100 & 4700 & 10000 & 6300 & 550 & 20000 & 290 & 4700 & 13000 & 180000 \\
\hline 1300 & 1900 & 440 & 480 & 38 & 290 & 1200 & 260 & 780 & 79 & 20 & 1300 & 140 & 34000 \\
\hline 1400 & 3700 & 30 & 150 & 120 & 950 & 640 & 160 & 540 & 800 & 120 & & 4800 & 47000 \\
\hline 6300 & 5000 & 4400 & 5900 & 8700 & 1400 & 2300 & 2500 & 1100 & - & 99 & 11000 & 2600 & 140000 \\
\hline 10000 & 5300 & 1800 & 1900 & 8200 & 260 & 1600 & 170 & 1400 & 250 & 200 & - & 3500 & 55000 \\
\hline 3300 & 1500 & 820 & 590 & 330 & 210 & 2100 & 1300 & 710 & 460 & 260 & 5300 & 5100 & 68000 \\
\hline 2700 & 5600 & 410 & 6000 & 740 & 5700 & 5100 & 770 & 700 & 20 & 98 & 8200 & 3100 & 120000 \\
\hline 7400 & 11000 & 3000 & 9300 & 870 & 3500 & 4100 & 2800 & 1300 & - & 260 & 39000 & 2600 & 220000 \\
\hline 50000 & 150000 & 62000 & 110000 & 75000 & 26000 & 100000 & 35000 & 32000 & 650000 & 5300 & 110000 & 220000 & 2900000 \\
\hline
\end{tabular}




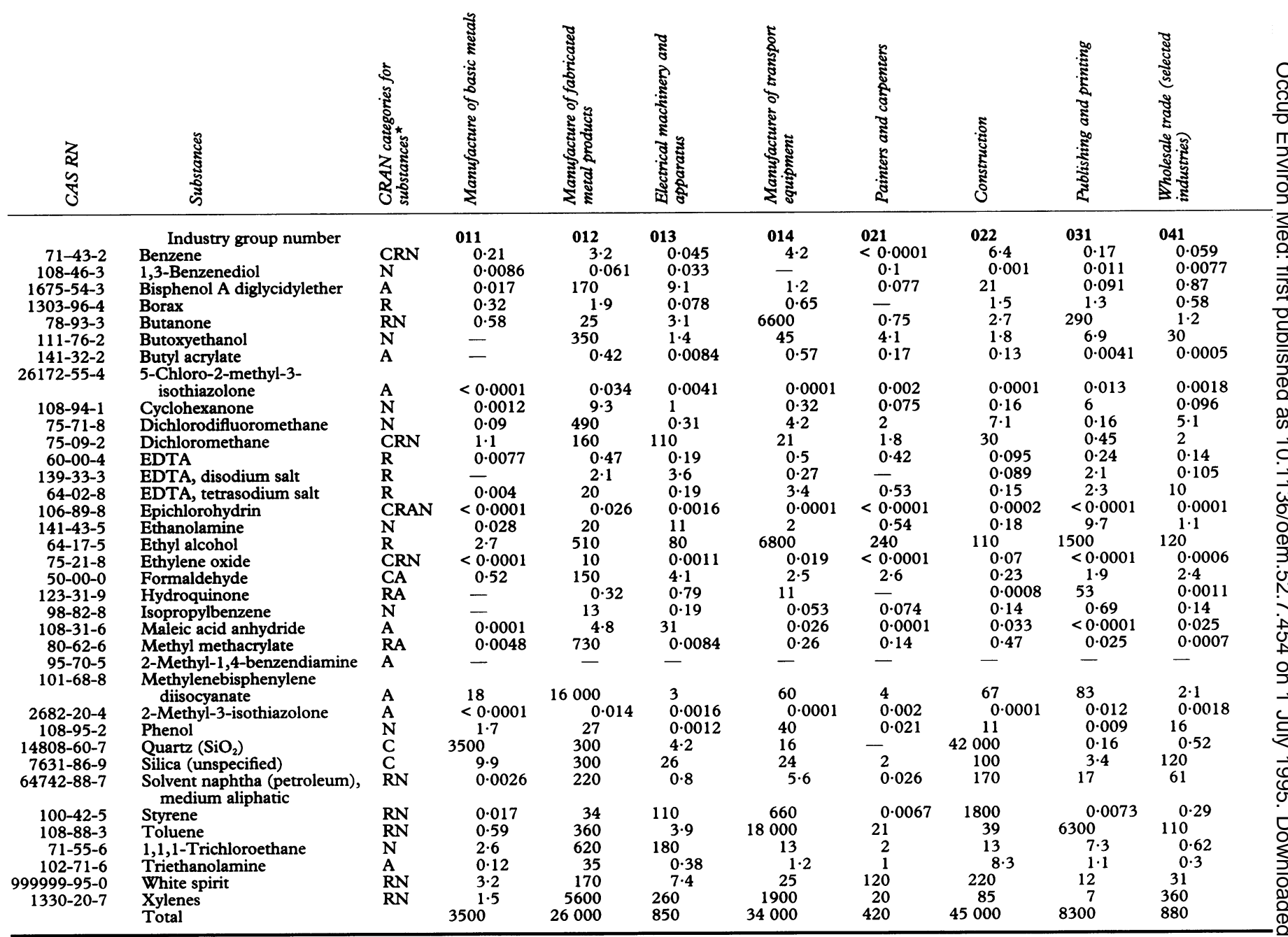

${ }^{\star} \mathrm{C}=$ carcinogenic; $\mathrm{R}=$ reproductivity toxicity; $\mathrm{A}=$ allergenic; $\mathrm{N}=$ neurotoxic.

ESTIMATED WEIGHTS

The estimated weights for the selected substances varied from a total of less than $50 \mathrm{~kg}$ to more than 200000 tonnes (table 2). High weight chemicals were silica (unspecified)* with 219000 tonnes occurring mainly in manufacture of non-metallic mineral products, and quartz $\left(\mathrm{SiO}_{2}\right)$ (14808-60-7) with 49000 tonnes occurring mainly in construction. Solvents represented a total of about 100000 tonnes, and typical high weight solvents were white spirit (999999-95-0) with 40000 tonnes occurring in manufacture of paints, petroleum, and bitumen products; toluene (108-88-3) with 26000 tonnes occurring in manufacture of transport equipment and in publishing and printing; xylenes with 16000 tonnes occurring in manufacture of paints, petroleum, and bitumen products, and in manufacture of fabricated metal products; ethyl alcohol with 13000 tonnes occur-

^Many registrations of products containing silica included crystalline and amorphous silica, as well as sand. Hence estimated weight will include non-carcinogenic silica, and consequently be much too high in this paper. ring in manufacture of transport equipment; butanone (78-93-3) with 6900 tonnes occurring in manufacture of transport equipment; methylenebisphenylene diisocyanate (MDI) (101-68-8) with 26000 tonnes occurring in manufacture of fabricated metal products and in manufacture of plastic and boat building and repair.

In the whole data set, disregarding the number of exposure events, high weights (>1000 tonnes) were estimated for 25 of the substances. For 11 of these, the estimated national number of total exposure events was less than 25000 .

In our paper table 2 presents estimated weights of substances with 25000 or more exposure events. The annual weights of use of chronic toxic chemicals are published in full length tables in a Danish report. ${ }^{13}$

For the carcinogens the highest estimated weights were found in manufacture of nonmetallic mineral products, construction, and manufacture of plastic and boat building and repair, but the highest estimated numbers of exposure events were found in manufacture of fabricated metal products, personal services, cleaning, and hair dressing, and construction. 


\begin{tabular}{|c|c|c|c|c|c|c|c|c|c|c|c|}
\hline 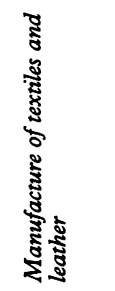 & 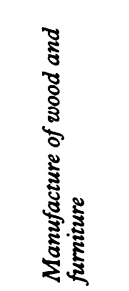 & 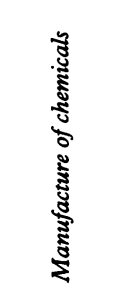 & 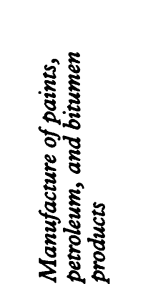 & 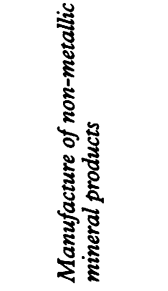 & 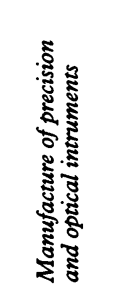 & 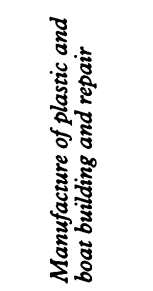 & 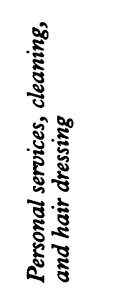 & 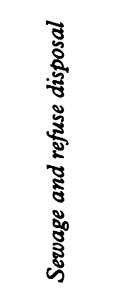 & 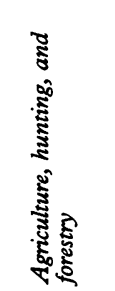 & 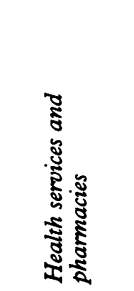 & \begin{tabular}{l}
$\bar{\Xi}$ \\
\multirow{0}{*}{}
\end{tabular} \\
\hline $\begin{array}{l}051 \\
0.0002 \\
- \\
0.02 \\
2 \cdot 4 \\
1 \cdot 1 \\
18 \\
0.0001\end{array}$ & $\begin{array}{l}052 \\
0.015 \\
- \\
7 \cdot 3 \\
0 \cdot 1 \\
32 \\
66 \\
0.0033\end{array}$ & $\begin{array}{c}053 \\
0.21 \\
0.035 \\
- \\
170 \\
0.13 \\
210 \\
0.019\end{array}$ & $\begin{array}{l}054 \\
0.012 \\
- \\
- \\
- \\
6.2 \\
7.5\end{array}$ & $\begin{array}{l}055 \\
\quad 5 \cdot 4 \\
0 \cdot 0084 \\
8.9 \\
0.13 \\
5 \cdot 8 \\
1.5 \\
0.0002\end{array}$ & $\begin{array}{l}056 \\
0.0034 \\
\frac{19}{0.29} \\
1.3 \\
0.061 \\
0.0003\end{array}$ & $\begin{array}{l}057 \\
\quad 0.0004 \\
- \\
0.63 \\
0.018 \\
3.4 \\
0.76 \\
-\end{array}$ & $\begin{array}{l}081 \\
0.0007 \\
0.017 \\
\overline{0.12} \\
<0.0001 \\
0.48 \\
0.0037\end{array}$ & $\begin{array}{l}082 \\
0.0003 \\
- \\
\overline{0} .046 \\
0.0035 \\
0.094 \\
-\end{array}$ & $\begin{array}{l}101 \\
0.007 \\
- \\
\overline{33} \\
0.026 \\
0.063 \\
0.012\end{array}$ & $\begin{array}{l}111 \\
\quad 0.12 \\
0.0065 \\
0.002 \\
0.028 \\
0.2 \\
13 \\
0.0025\end{array}$ & $\begin{array}{c}20 \\
0 \cdot 29 \\
240 \\
210 \\
6900 \\
750 \\
8 \cdot 8\end{array}$ \\
\hline $\begin{array}{l}0.04 \\
0 \cdot 16 \\
0.17 \\
0.006 \\
0.066 \\
0.0025 \\
36 \\
<0.0001 \\
47 \\
6.9 \\
0.0083 \\
4 \cdot 6 \\
0.0001 \\
0.0008 \\
0.026 \\
-\end{array}$ & $\begin{array}{l}1.5 \\
0.12 \\
1 \\
6.9 \\
0.13 \\
0.042 \\
100 \\
\quad 0.0007 \\
0.091 \\
650 \\
<0.0001 \\
30 \\
<0.0001 \\
0.29 \\
0.14 \\
0.031 \\
-\end{array}$ & $\begin{array}{c}0.43 \\
4 \cdot 1 \\
\overline{55} \\
380 \\
\frac{1}{2600} \\
\overline{1}-1 \\
1300 \\
0 \cdot 0042 \\
4 \cdot 9 \\
0 \cdot 0023 \\
4 \cdot 1 \\
0 \cdot 0004 \\
<0 \cdot 0001 \\
-\end{array}$ & $\begin{array}{l}0.0013 \\
0.038 \\
\overline{86} \\
- \\
\overline{0} \\
0 \cdot 12 \\
0.011 \\
0 \cdot 1 \\
990 \\
\frac{0}{0.077} \\
\frac{17}{34} \\
12 \\
-\end{array}$ & $\begin{array}{c}0.0001 \\
0.074 \\
0.12 \\
43 \\
0.081 \\
0.75 \\
1.9 \\
0.0024 \\
0.0075 \\
81 \\
0.0032 \\
4300 \\
<0.0001 \\
0.12 \\
0.0056 \\
0.0017 \\
-\end{array}$ & $\begin{array}{l}0.0005 \\
0.6 \\
0.073 \\
19 \\
0.0051 \\
0.0001 \\
0.051 \\
0.0044 \\
\overline{46} \\
<0.0001 \\
0.0001 \\
0.6 \\
<0.0001 \\
<0.0001 \\
0.0045 \\
-\end{array}$ & $\begin{array}{c}0.048 \\
0.28 \\
5.8 \\
1200 \\
0.57 \\
0.01 \\
0.96 \\
<0.0001 \\
0.047 \\
24 \\
12 \\
0.1 \\
1.3 \\
0.047 \\
0.26 \\
0.026 \\
-\end{array}$ & $\begin{array}{l}0.0027 \\
\frac{0.062}{3} \\
3.5 \\
0.001 \\
\frac{33}{20} \\
64 \\
0.0001 \\
0.1 \\
0.0024 \\
\frac{-}{\overline{0}} \\
0.0037 \\
0.044\end{array}$ & $\begin{array}{l}<0.0001 \\
\overline{-} \\
\overline{0} \\
\overline{0} .12 \\
\overline{0} .19 \\
\overline{-} \\
\frac{0}{0} .93 \\
\frac{0}{<0018} \\
<0.0001 \\
\frac{0}{0} .01\end{array}$ & $\begin{array}{l}0.0006 \\
\mathbf{4 4} \\
\mathbf{4} \cdot 6 \\
0.005 \\
0.21 \\
0.001 \\
0.015 \\
-1 \\
\mathbf{4} \cdot 5 \\
0.0002 \\
0.62 \\
0.0003 \\
0.68 \\
-0.012 \\
-\end{array}$ & $\begin{array}{l}0.0004 \\
0.0005 \\
0.35 \\
0.66 \\
0.65 \\
0.0085 \\
39 \\
- \\
0.76 \\
210 \\
<0.0001 \\
5.9 \\
3.3 \\
0.0002 \\
<0.0001 \\
16 \\
-\end{array}$ & $\begin{array}{c}2 \\
66 \\
520 \\
1700 \\
390 \\
9 \cdot 1 \\
2900 \\
0 \cdot 047 \\
280 \\
13000 \\
23 \\
4500 \\
71 \\
36 \\
71 \\
760 \\
0.044\end{array}$ \\
\hline $\begin{array}{l}- \\
0.038 \\
1 \\
0 \cdot 009 \\
6 \cdot 3 \\
0 \cdot 86\end{array}$ & $\begin{array}{c}1.4 \\
0.55 \\
1.2 \\
0.038 \\
180 \\
3.1\end{array}$ & $\begin{array}{c}- \\
0.16 \\
0.85 \\
0.38 \\
120 \\
0.0001\end{array}$ & $\begin{array}{c}\overline{0} .0005 \\
\overline{3} \cdot 7 \\
1200 \\
0.47\end{array}$ & $\begin{array}{c}240 \\
<0.0001 \\
4100 \\
2700 \\
216000 \\
5\end{array}$ & $\begin{array}{c}130 \\
0.0005 \\
0.0023 \\
0.01 \\
26 \\
0.5\end{array}$ & $\begin{array}{c}9700 \\
0.0014 \\
<0.0001 \\
1.6 \\
36 \\
0.37\end{array}$ & $\begin{array}{l}- \\
0.001 \\
0.0002 \\
0.32 \\
0.012 \\
2 \cdot 2\end{array}$ & $\begin{array}{l}<\overline{0} .0001 \\
0.0009 \\
\overline{0} \\
0.22 \\
0.057\end{array}$ & $\begin{array}{l}- \\
0.0006 \\
0.65 \\
0.13 \\
5.6 \\
6.1\end{array}$ & $\begin{array}{l}0.36 \\
0.0002 \\
0 \cdot 25 \\
6 \cdot 4 \\
3 \cdot 1 \\
0 \cdot 068\end{array}$ & $\begin{array}{c}26000 \\
0 \cdot 78 \\
4100 \\
49000 \\
219000 \\
490\end{array}$ \\
\hline $\begin{array}{c}0 \cdot 052 \\
15 \\
1 \cdot 1 \\
0 \cdot 44 \\
140 \\
14 \\
300\end{array}$ & $\begin{array}{c}0 \cdot 064 \\
420 \\
97 \\
0 \cdot 16 \\
5300 \\
920 \\
7800\end{array}$ & $\begin{array}{c}1 \cdot 4 \\
170 \\
2 \\
530 \\
730 \\
11 \\
6500\end{array}$ & $\begin{array}{c}1 \cdot 3 \\
420 \\
0 \cdot 53 \\
0.91 \\
32000 \\
6600 \\
41000\end{array}$ & $\begin{array}{c}0 \cdot 28 \\
17 \\
55 \\
68 \\
81 \\
44 \\
228000\end{array}$ & $\begin{array}{c}16 \\
19 \\
2 \cdot 3 \\
0 \cdot 12 \\
3 \cdot 8 \\
4 \cdot 8 \\
290\end{array}$ & $\begin{array}{c}38 \\
0 \cdot 62 \\
700 \\
110 \\
17 \\
140 \\
12000\end{array}$ & $\begin{array}{l}\quad 0.0056 \\
\quad 0.068 \\
7 \\
0.21 \\
\quad 5 \\
0.025 \\
140\end{array}$ & $\begin{array}{l}0.033 \\
0.076 \\
0.057 \\
0 \cdot 11 \\
8 \cdot 9 \\
6 \cdot 1 \\
17\end{array}$ & $\begin{array}{c}\overline{0} \\
\overline{0} \\
0 \cdot 74 \\
28 \\
190 \\
320\end{array}$ & $\begin{array}{c}0.0035 \\
4 \cdot 1 \\
1 \cdot 05 \\
0 \cdot 99 \\
1200 \\
5 \cdot 3 \\
1500\end{array}$ & $\begin{array}{r}2700 \\
26000 \\
1700 \\
760 \\
40000 \\
16000 \\
-\end{array}$ \\
\hline
\end{tabular}

For the reproductive toxicants the highest estimated weights were found in manufacture of paints, petroleum, and bitumen products, transport equipment, and fabricated metal products, and publishing and printing, but the highest estimated numbers of exposure events were found in manufacture of fabricated metal products, personal services, cleaning, and hair dressing, and health services and pharmacies.

For the allergens the highest estimated weights were found in manufacture of fabricated metal products, and manufacture of plastic and boat building and repair, but the highest estimated numbers of exposure events were found in manufacture of fabricated metal products, and personal services, cleaning, and hair dressing.

For the neurotoxicants the highest estimated weights were found in manufacture of paints, petroleum, and bitumen products, manufacture of transport equipment, and manufacture of fabricated metal products, but the highest estimated numbers of exposure events were found in manufacture of fabricated metal products, and personal services, cleaning, and hair dressing.
NUMBERS OF DIFFERENT PRODUCTS REGISTERED

The numbers of different products registered for the 36 substances (with a total of 25000 or more estimated exposure events) were between 27 and 775 . The substances with the highest estimated numbers of different products registered were xylenes (775 products) occurring mainly in manufacture of fabricated metal products, in electrical machinery and apparatus, and in construction; silica (unspecified) (594 products) occurring mainly in manufacture of fabricated metal products and in electrical machinery and apparatus; ethyl alcohol (491 products) occurring mainly in publishing and printing, in manufacture of fabricated metal products, and in health services and pharmacies; toluene (473 products) occurring mainly in manufacture of fabricated metal products and in electrical machinery and apparatus; white spirit (414 products) occurring mainly in manufacture of fabricated metal products; and formaldehyde (50-00-0) ( 372 products) occurring mainly in manufacture of fabricated metal products.

In the whole data set, disregarding the number of exposure events, a total of 28 
substances were registered in more than 100 different products. For three of these the estimated number of exposure events was less than 25000 .

\section{Discussion}

High numbers of exposure events in the industry groups included were estimated for some preservatives, solvents, and unspecified silica. Not all chemicals with many estimated exposure events were high weight chemicals, and several high weight chemicals did not have high estimated numbers of exposure events. Industries that merit special attention as assessed by the estimated national number of exposure events were manufacture of fabricated metal products, and personal services, cleaning, and hair dressing.

The validity of the national estimates of the number of exposure events and of the annual use (weights) of chemicals is dependent on the representativeness of the businesses that were included in the sample and on their response to the questionnaire. The validity of the response information was controlled in the coding and registration phase of the survey, and considered good. Businesses answered according to instructions or consultations, and telephone questionnaires were used to specify ambiguous answers, along with random work environment service inspections of work sites (no statistical validity control was performed). As the representative nature and the proportion of products with information on composition varied between the 19 industry groups, the data for some industry groups should be considered more valid than for others. For the total investigation answers were obtained for $70 \%$ of the 1448 businesses, information on composition were obtained for $73 \%$ of the products, and information on weights for $88 \%$. The industry groups with the highest national estimates of the number of exposure events (manufacture of fabricated metal products, personal services, cleaning, and hair dressing, and health services and pharmacies) had response rates from the businesses of $74 \%, 83 \%$, and $83 \%$ respectively, information on composition of the products of $76 \%, 84 \%$, and $78 \%$ respectively, and information on weights of $88 \%$, $92 \%$, and $90 \%$ respectively. Therefore, the validity of these data seems satisfactory.

From data to be published it seems that each industry group has its own characteristic set of work processes comprising the major use of chemicals. For each industry group the reported work processes were concentrated on six to eight processes out of the 20-50 defined for each industry group, which supports the assumption of homogeneity within each group.

Manufacture of fabricated metal products had the highest estimates of the number of exposure events for 18 of the 36 substances and the highest numbers of registered products for 27 of the 36 substances. This industry group also has the highest number of employees. It is suggested that the high estimated number of exposure events is due to the use of many different metal working and cutting fluids, degreasing agents, paints, metal coatings, etc that contain many different toxic substances. Especially, metal working and cutting fluids are more often registered in the present investigation than in a previous study. ${ }^{16}$

Another industry group with a very high national estimate of the number of exposure events was personal services, cleaning, and hair dressing although it has fewer employees. A few substances (1,3-benzendiol (108-46-3) and ethanolamine) were used in many different products within this industry group, which again contributes to the high estimate of the number of exposure events. Health services and pharmacies and construction also showed high national estimates of the number of exposure events in agreement with their high number of employees.

Manufacture of fabricated metal products and manufacture of chemicals had the highest national loads of chemicals-based on the estimated total weights of the chronic toxic substances included. Users of silica (unspecified) as sand had the highest weights of usefor example, manufacture of non-metallic mineral products that used 216000 tonnes of silica. Industries with medium weights were manufacture of paints, petroleum, and bitumen products with an estimated weight of 32000 tonnes of white spirit, and manufacture of transport equipment with 18000 tonnes of toluene.

Previous publications on registered chemicals have ranked chemicals by the number of different products containing the substances. ${ }^{16} 17$ In our presentation, the main criterion for ranking was the estimated number of exposure events. For the selected substances the estimated weights used and the number of different products registered were evaluated. Xylenes, silica, toluene, and ethyl alcohol were high on all three exposure measures. Within industry groups this tendency was less pronounced although manufacture of fabricated metal products was high both for substances with a high number of registered products and for a high estimated number of exposure events.

A questionnaire based survey of the working conditions of employees in Denmark was conducted in $1990 .{ }^{18}$ The exposure to chemicals reported by the employees agreed with the results of our survey: exposure to chemical products was considered a major problem in cleaning, social and health services (cleaning agents), and in the construction and the wood and furniture industry (solvents). ${ }^{19}$

In contrast with trade statistics on chemicals, our study includes substances that occur as components in the products-for example, solvents as part of paints, which will be missing when the import of pure substances (raw materials) and of the formulated products are registered. Hence, the national weights for the industry groups included in this study will be higher and more valid than trade statistics. To illustrate this the annual use of white spirit amounted to 19400 tonnes according to 
trade statistics and 40000 tonnes in this study of selected industry groups from the same year. ${ }^{20} 21$ Also, this study includes information on the industrial areas of use.

A Danish investigation of a stratified random sample of businesses in the wood and furniture industry included a survey of the use of chemical products. In this survey 673 different chemical products were found in 200 businesses. ${ }^{22}$ In our investigation 645 different chemical products were registered in the wood and furniture industry group, but the data were collected from fewer businesses. Most of the frequently registered substances in our investigation were included among the 20 most frequent substances in the earlier survey, which implies that a representative sample had been drawn in this industry group.

For carcinogens, the estimated weights used may be compared with a registration based on mandatory notifications from Danish manufacturers and importers. ${ }^{23}$ Comparisons for about 40 selected carcinogens showed that the annual use was lower in our study for $80 \%$ of these substances. Twenty per cent were more than 100 times lower. Some of the industrial areas excluded in our investigation may have contributed in the notification of carcinogens - for example, public and private transportation, manufacture of food and drink, etc. The comparison was difficult because the notification data did not specify industry groups for all chemicals. According to the notification of carcinogens, ${ }^{23}$ 127 of 240 carcinogens (53\%) listed in 1989 were used in Denmark, and for our investigation 73 of 284 carcinogens (26\%) listed in 1992 were found in the reported products. This discrepancy may be due both to the excluded industrial areas and to the fact that information on components was missing for a quarter of the products in our investigation.

Occurrence of contact allergens has mainly been investigated with a focus on product categories. ${ }^{16} \mathrm{~A}$ comparison shows that among the 10 most often registered contact allergens in an investigation made in 1990, five were among the most frequent allergens in the present investigation-for example, epichlorohydrin (106-89-8), bisphenol A diglycidylether (1675-54-3), formaldehyde, methylenebisphenylene diisocyanate, and triethanolamine (102-71-6). Substances occurring in the industry group personal services, cleaning, and hair dressing may be compared with substances found in an investigation based on registration of all cleaning agents used in Denmark in 1986 and still marketed in $1992 .{ }^{24}$ For the allergens included in both the investigation on cleaning agents and in our investigation, the most often registered substances were the same-namely, the active ingredients in Kathon CG (5-chloro-2-methyl3-isothiazolone and 2-methyl-3-isothiazolone), formaldehyde, and triethanolamine.

The model of extrapolation assumes that the groups of businesses are comparable in their use of chemicals. Homogeneity may not be the case, due to the size of the samples and the number of groups in the study being limited to $19 \times 4=76$. Although the use of chemicals may be well evaluated qualitatively, some indications of deficiencies in the quantitative aspects of use are present. Compared with statistics for the annual supply of pesticides, ${ }^{25}$ our investigation tends to underestimate the use of specific pesticides (less than five times for half of the 15 pesticides included in our data, and several thousand times for two of the pesticides). The same tendency was seen in comparison with data on carcinogens registered by notifications from the manufacturers and importers (already mentioned). The estimation of annual use will, however, be further studied, and other models may be considered. For each industrial area further investigation may show different methods of extrapolation, and the model used may prove valid for selected areas only.

For chemicals with equally toxic properties and equal risk the choice of preventive measures depends on the distribution of the chemical in focus. When the number of exposure events is high, substitution may be the choice to prevent hazardous exposure, and when the number of exposure events is low or concentrated on few businesses it may be encapsulation or ventilation.

Two investigations have been used for setting priorities ${ }^{2}$ and for development of a job exposure matrix..$^{26}$ These surveys on the use of chemical products were performed in the United States in 1972 and in 1980. The 1972 national occupational hazard survey included a sample of 4636 businesses. About 86000 different trade named products were identified. Satisfactory component information was obtained for about $81 \%$ of the products. ${ }^{1}$ In 1980 the national occupational exposure survey was started, which included 4490 businesses. More than 100000 different trade named products were recorded. In June 1988 component information was obtained for about $50 \%$ of the products and the data were still regarded as provisional. ${ }^{2}$ In our Danish survey component information was obtained for about $73 \%$ of the products when the data collection was closed after two years.

Surveys that rely only on data on weights of chemicals used may be criticised on the ground that many high weight chemicals are processed with little or no human exposure (enclosed processes). As a unique feature, our study collected data on the number of people who work with the substances, and it seems that chemicals that are used in large quantities and entail many exposure events should be given most attention, other things being equal.

Still we do not have information on the intensity or duration of the exposure. Different technologies may entail different levels of exposure-for example, manual assembly processes with solvent based glue can entail higher exposure levels for the individual worker than solvent based paints applied in industrial painting plants although the weights of chemicals used are larger in the painting industry. Therefore, the numbers of estimated exposure events cannot be taken 
simply at face value when priorities are established. The combined information, however, on exposure events, weights, and other available knowledge (exposure, number of employees, preventive measures, etc) on the industry in question will reinforce the basis of the establishment of priorities.

This survey on the use of chemicals is the first nationwide investigation in Denmark to delineate the use of all chemicals (pure substances or components in chemical products). The investigation has provided a background knowledge of exposure to chemicals. In countries like Denmark (without a large production of (pure) chemical compounds or substances) this type of survey may be very useful as there is a need for knowledge on hidden occupational exposure to substances included in formulated products.

The study as a whole has been used in a project to review occupational hazards in general in Danish industry. ${ }^{27}$ It will be a basis for measuring chemical substitution, development of chemical safety, and a reference data set for comparison in more specific investigations and follow up studies.

As information on work processes is included in the investigation, job exposure matrices based on the actual use of chemical products can be constructed from the collected data. Experiences from other studies will be used in future work and if possible the documentation and sample programmes available from the job exposure matrices based on the national occupational hazard survey will be adopted. ${ }^{26}$

Further surveys will monitor the development of chemical exposures in the working environment and the use of chemicals hazardous to health and to the external environment.

The design of our survey is planned to be used in another survey of a selected industry-namely, the printing industry. This study focuses on the use of carcinogens.

We acknowledge staff members in all parts of the working environment service involved in the data collection for ou investigation.

\footnotetext{
Appendix: Industrial categories and ISIC codes included in each industry group

A special grouping of industrial areas was made for our chemical survey to cover groups with an expected comparable use of chemicals. The industry group numbers (the first two digits) refer to the Danish Sector Safety Council No, meaning that group 011, 012, 013, and 014 belong to the Sector Safety Council No 1.

INDUSTRY GROUP 011: MANUFACTURE OF BASIC METALS ISIC 37XX Basic metal industries

INDUSTRY GROUP 012: MANUFACTURE OF FABRICATED METAL PRODUCTS

ISIC 381X Manufacture of fabricated metal products

ISIC 382X Manufacture of machinery and equipment

ISIC 41XX Electricity, gas, and steam

ISIC 42XX Water works and supply

ISIC 9512 Electrical repair shops

ISIC 9513 Repair of motor vehicles and motorcycles

ISIC 9514 Watch, clock, and jewellery repair

ISIC 9515 Repair and services of bicycles
}

ISIC 9519 Other repair not elsewhere classified (nec) INDUSTRY GROUP 013: ELECTRICAL MACHINERY AND APPARATUS

ISIC 383X Manufacture of electrical machinery, apparatus, appliances, and supplies

INDUSTRY GROUP 014: MANUFACTURE OF TRANSPORT EQUIPMENT

ISIC 38YX (excluding boat building and repair) manufacture of transport equipment

INDUSTRY GROUP 021: PAINTERS AND CARPENTERS ISIC 5014 Carpenters and joiners

ISIC 5015 Painters

INDUSTRY GROUP 022: CONSTRUCTION

ISIC 50XX (excluding carpenters, joiners, and painters) construction

INDUSTRY GROUP 031: PUBLISHING AND PRINTING

ISIC 3412 Manufacture of containers and boxes of paper and paperboard

ISIC 3419 (excluding manufacture of wall paper) manufacture of pulp, paper, and paper board articles ISIC 342X Printing, publishing, and allied industries ISIC 8325 Advertising services

INDUSTRY GROUP 041: WHOLESALE TRADE (SELECTED INDUSTRIES)

Specific Danish subdivision of ISIC 6116,6118 , and 6119:

Grain, feeding stuffs, and fertilisers (subdivision of ISIC 6116)

Seeds and bulbs (subdivision of ISIC 6116)

Timber, and building materials (subdivision of ISIC 6116)

Machinery and equipment (subdivision of ISIC 6118)

Motor cars, motorcycles and parts, petrol, solid and liquid fuels, lubricants (subdivision of ISIC 6119)

INDUSTRY GROUP 051: MANUFACTURE OF TEXTILES AND LEATHER

ISIC 32XX Textile, wearing apparel, and leather industries

ISIC 9511 Repair of footwear and leather goods

INDUSTRY GROUP 052: MANUFACTURE OF WOOD AND FURNITURE

ISIC 33XX (excluding upholstered furniture) manufacture of wood and wood products

ISIC 3411 Manufacture of pulp, paper, and paperboard

ISIC 3410 (part) Manufacture of wall paper

INDUSTRY GROUP 053: MANUFACTURE OF CHEMICALS ISIC 3511 Manufacture of basic industrial chemicals except fertilisers

ISIC 3512 Manufacture of fertilisers and pesticides

ISIC 3522 Manufacture of drugs and medicines ISIC 3523 Manufacture of soaps and cosmetics ISIC 3529 Manufacture of chemicals, nec

ISIC 355X Manufacture of rubber products

INDUSTRY GROUP 054: MANUFACTURE OF PAINTS, PETROLEUM, AND BITUMEN PRODUCTS

ISIC 3521 Manufacture of paints, varnishes, and lacquers

ISIC 3530 Petroleum refineries

ISIC 354X Manufacture of miscellaneous products of petroleum and coal

INDUSTRY GROUP 055: MANUFACTURE OF NONMETALLIC MINERAL PRODUCTS

ISIC 36XX Manufacture of non-metallic mineral products except petroleum and coal

INDUSTRY GROUP 056: MANUFACTURE OF PRECISION AND OPTICAL INSTRUMENTS

ISIC 3851 Manufacture of professional and scientific, and measuring and controlling equipment, nec (excluding orthopaedic and prosthetic appliances) 
ISIC 3852 Manufacture of photographic and optical goods

ISIC 39XX Other manufacturing industries

ISIC $62 \mathrm{XX}$ (part of wholesale trade, excluding photographic equipment) retail trade of clocks, jewellery and pewter, and optical goods

INDUSTRY GROUP 057: MANUFACTURE OF PLASTIC AND BOAT BUILDING AND REPAIR

ISIC 3513 Manufacture of synthetic resins, plastic materials, and man made fibres except glass ISIC 3560 Manufacture of plastic products, nec ISIC 3841 (part) Boat building and repair

INDUSTRY GROUP 081: PERSONAL SERVICES, CLEANING AND HAIR DRESSING

ISIC 9202 (part) House and window cleaning ISIC 9520 Laundries, laundry services, and cleaning and dyeing plants

ISIC 9591 Barber and beauty shops

INDUSTRY GROUP 082: SEWAGE AND REFUSE DISPOSAL ISIC 9200 (part) Garbage and sewage disposal

INDUSTRY GROUP 101: AGRICULTURE, HUNTING, AND FORESTRY

ISIC 11XX Agriculture and hunting

ISIC 12XX Forestry and logging

INDUSTRY GROUP 111: HEALTH SERVICES AND PHARMACIES

ISIC 3851 (part) Manufacture of orthopaedic and prosthetic appliances

ISIC 62XX (part) Pharmacies

ISIC 9331 Medical, dental, and other health services

ISIC 9332 Veterinary services

1 Sundin DS. The national occupational hazard survey: a difficult quest for a reliable data base. Occup Health Saf 1978;47:21-3

2 Sundin DS, Frazier TM. VII. Hazard surveillance at NIOSH. Am $₹$ Public Health 1989;79:32-7.

3 Flyvholm M-A, Andersen P, Beck ID, Brandorff NP. PROBAS: the Danish product register data base-a national register of chemical substances and products. fournal of Hazardous Materials 1992;30:59-69.

4 National Institute of Occupational Health. Action programme for research on carcinogens, reprotoxicants, allergens, gramme for research on carcinogens, reprotoxicants, allergens, and neurotoxicants. Copenhagen: Nation

5 Danish National Labour Inspection. Registration of substances and materials considered to be carcinogenic. Copenhagen: Danish National Labour Inspection, 1990. (Order No 535.) 6 Hass U, Jacobsen BM, Brandorff NP, Jelnes JE, Petersen
SH. Reproductive toxicants in the working environmentnt. Danish National Institute of Occupational Health, 1991. (AMI-report No 35.) (In Danish; summary in English.)

7 Danish Environmental Protection Agency. List of dangerous substances. Copenhagen: Danish Environmental Protection Agency, 1991. (Order No 589.)
8 Kemikalieinspektionen. List of allergenic substances, 1 fuly 1986; changes from February 1990. Stockholm: 1986; changes from February

9 Statens Forurensningstilsyn. List of dangerous substances, 19 fanuary 1983; changes 29 April 1986. Oslo: Statens Forurensningstilsyn, 1986.

10 Social- och hälsovảrdsministeriet. Order on classification and labelling of dangerous substances, 21 December 1988. Helsingfors: Social- och häsovårdsministeriet, 1988.

11 Simonsen L, Lund SP. A strategy for delineating risks due to exposure to neurotoxic chemicals. $\mathrm{Am}$ f Ind Med 1992;21:773-92.

12 Zebitz U, Brandorff NP. A national survey on use of chemicals in Danish enterprises 1989. Background, purpose, and data collection. Copenhagen: National Institute of Occupational Health, 1991. (In Danish.)

13 Brandorff NP, Beck ID, Skov T, Flyvholm M-A. Use of chemicals and potential chemical exposures in Danish enterprises 1989. A national survey. Copenhagen: National Institute of Occupational Health, 1994. (AMI-report No 43.) (In Danish; summary in English.)

14 Statistical Office of the United Nations. Indexes to the international standard industrial classification of all economic activities. Statistical papers. New York: United Nations, 1971. (Series M No 4, REV 2, ADD 1.)

15 Børglum B, Hansen AM. A survey of washing and cleaning agents. Copenhagen: National Institute of Occupational agents. Copenhagen: National Institute of Occupational mary in English.)

16 Flyvholm M-A. Contact allergens in registered chemical products. Contact Dermatitis 1991;25:49-56.

17 Seedorff L, Olsen E. Exposure to organic solvents-I. A survey on the use of solvents. Ann Occup Hyg 1990; 34:371-8

18 Ørhede E. Monitoring of the working environment in Denmark and in other nordic countries. In: Convergence des Modelles Sociaux Europeens. $4^{\text {ime }}$ séminaire sur l'Europe sociale. Paris 8 et 9 Octobre 1992. Rapport. Paris: Services des Études et de la Statistique, 1992. 173-7.

19 Ørhede E, Nord-Larsen M, Burr H, Nielsen J. Working environment in Denmark 1990 vol 2. Survey on exposure, work-processes, and health. Copenhagen: The Danish Working Environment Fund, 1992. (In Danish.)

20 Danmarks Statistik (National Bureau of Statistics). Foreign trade divided into products and countries. Statistical service. trade divided into products and countries. Statistical sert

21 Danmarks Statistik (National Bureau of Statistics). Product statistics for industry. Series B. Mineral and chemical products, wood and paper and goods from this. Statistical service. Copenhagen: National Bureau of Statistics, 1990.

22 Børglum B, Damgaard K, Nielsen S. Wood- and furniture industry. Cross sectional survey chemical substances and products. Copenhagen: The Labour Inspection, 1989. (At Report No 7.) (In Danish.)

23 Bjersing M, Hansen J, Schöller C, Thomsen P. Carcinogens and potential carcinogens in Denmark - the distribution and consumption of 240 substances in 1989. Copenhagen: National Institute of Occupational Health, 1992. (AMIreport No 37.) (In Danish; summary in English.)

24 Flywholm M-A. Contact allergens in registered cleaning agents for industrial and household use. $\mathrm{Br} \mathcal{f}$ Ind $\mathrm{Med}$ 1993;50:1043-50.

25 Danish Environmental Protection Agency. Sales of pesticides 1987, 1988, and 1989. Copenhagen: Danish Environmental Protection Agency, 1990. (In Danish.)

26 Sieber WK Jr, Sundin DS, Frazier TM, Robinson CF Development, use, and availability of a job exposure matrix based on national occupational hazard survey data. Am F Ind Med 1991;20:163-74.

27 Working Environment Service. Trade profiles. $A$ valuable aid in work health and safety activities. Copenhagen: Working Environment Service, 1993. 